Article

\title{
A Model-Free Diagnosis Approach for Intake Leakage Detection and Characterization in Diesel Engines
}

\author{
Ghaleb Hoblos ${ }^{\dagger} * *$ and Mourad Benkaci ${ }^{\dagger}$ \\ ESIGELEC-IRSEEM, Avenue Galilée, 76801 Saint Etienne du Rouvray, France; \\ E-Mail: ghaleb.hoblos@esigelec.fr \\ $\dagger$ These authors contributed equally to this work. \\ * Author to whom correspondence should be addressed; E-Mail: ghaleb.hoblos@esigelec.fr; \\ Tel.: +33-2-32-91-58-58; Fax: +33-2-32-91-58-59.
}

Academic Editor: David Mba

Received: 2 October 2014 / Accepted: 21 July 2015 / Published: 31 July 2015

\begin{abstract}
Feature selection is an essential step for data classification used in fault detection and diagnosis processes. In this work, a new approach is proposed, which combines a feature selection algorithm and a neural network tool for leak detection and characterization tasks in diesel engine air paths. The Chi square classifier is used as the feature selection algorithm and the neural network based on Levenberg-Marquardt is used in system behavior modeling. The obtained neural network is used for leak detection and characterization. The model is learned and validated using data generated by xMOD. This tool is used again for testing. The effectiveness of the proposed approach is illustrated in simulation when the system operates on a low speed/load and the considered leak affecting the air path is very small.
\end{abstract}

Keywords: leak detection; automotive diagnosis; feature selection; neural data classification; diesel air path

\section{Introduction}

In order to reduce air pollution caused by automobile engines, several standards have been introduced. The first standard was proposed by the California Air Resources Board in 1970. Since 1993, marked by the introduction of the Kyoto Protocol, the European anti-pollution standards have become more stringent where the authorized emissions of a diesel vehicle have been decreased from (NOx = nil, 
$\mathrm{CO}=2720, \mathrm{HC}+\mathrm{NOx}=970, \mathrm{PM}=140)$ in Euro1 standard to $(\mathrm{NOx}=80, \mathrm{CO}=500, \mathrm{HC}+\mathrm{NOx}=170$, $\mathrm{PM}=5$ ) in Euro6 standard. Typically, each fault that increases the emission level must be detected and isolated. The leaks in the intake canal are among the most difficult faults to manage. Several works have been devoted to fault detection and isolation (FDI) in automotive applications [1-4]. According to the above-mentioned references, process knowledge used in the form of a mathematical process model enables model-based FDI approaches to enhance the performance of the engine diagnosis systems. Nyberg [1,2] used structured hypothesis testing for additive and multiplicative faults in production engines. A real-time observing method which uses observers that estimate unknown fault-parameters was discussed in [3]. In [4], structured parity equations are used to detect and isolate malfunctions in sensors and actuators.

The ability of neural networks to approximate a nonlinear function makes them one of the best tools for fault detection and isolation. By exploring these artificial intelligence techniques, researchers developed other classes of fault detection and isolation algorithms. In [5], Isermann discussed the superior features of neural networks in fault classification and recognition. Sorsa and Costin [6] show the capabilities of supervised neural networks such as Multilayers Perceptron (MLP) and Radial Basis Function (RBF) to perform good and effective fault detection and isolation tasks. Another work using MLP network is presented in [7]. The RBF was used in on-board fault diagnosis for the air path of spark ignition engines [8]. The leakage problem of gasoline engines is dealt with in [9] where the neural network based on the steepest-descent method combined with a back propagation algorithm is developed to train three detection systems.

Because of the increased complexity of today's engines, which are characterized by a large number of sensors, the reduction of the acquired data becomes essential.

Feature selection is one of important steps before beginning a data classification task, especially when this task is dedicated to fault detection and isolation. It refers to the problem of selecting the input features that are most predictive for a given outcome. The feature selection problems are found in all supervised and unsupervised machine learning which include classification, regression, time-series, prediction and clustering. The feature selection tasks try to achieve three main goals: reduce the cost of extracting features, improve the classification accuracy and enhance the reliability of the estimated performances.

There are many works that use the feature selection algorithm for fault detection and diagnosis. In reference [10], a new approach for intrusion detection and diagnosis is proposed. It combines the sequential backward floating search which selects the pertinent features with the fuzzy ARTMAP [11] classification used for detection and diagnosis of attacks. In this work, the vigilance parameter of fuzzy ARTMAP is fixed using a Genetic Algorithm (GA). In [12], the authors propose using a new optimization algorithm in the feature selection procedure. This algorithm is based on a modified binary particle swarm optimization with mutation combined with support vector machine classification. In [13] an improved distance evaluation technique is used to select the optimal features and the importance of features in diagnosis processes is pondered by weight. In reference [14], the authors use the decision tree to identify the best features in the classification task. They use a proximal support vector machine characterized by its capability to efficiently classify the faults in Roller Bearing systems. In [15], the authors perform a diagnosis of induction motors using pattern recognition methods. They use a sequential backward algorithm in order to select the most relevant features. In the previous work, the 
classification is assured by the k-nearest neighbors rule including reject options. The authors of [16] propose to incorporate a GA with Fisher Discriminant Analysis (FDA) in the key variables identification procedure. The GA is used to select the features that optimize the FDA classification success rate. This approach is applied to the data generated by the Tennessee Eastman Process (TEP) simulator. A new method for feature selection based on mutual information for fault detection and identification is proposed in [17]. Their algorithm is based on two principle stages: the variables are sorted based on their shared mutual information with the class variable and secondly the more informative variables are chosen by taking into account the classification error rate. Once more, the approach is applied in the TEP (Tennessee Eastman Process) simulator. In [18], the authors use a recursive feature elimination to select key variables using Support Vector Machines (SVM). The SVM is combined with time lags incorporated before every classification step. Finally, the number of relatively important variables determined by each classifier is basically determined by 10 -fold cross-validation. Wang [19] introduces a neural network approach to vibration feature selection in mechanical systems fault detection. He proposes an artificial intelligence methodology for mechanical fault detection using vibration data, which includes intelligent feature optimization. He uses a back-propagation neural network twice, the first one for feature selection and the second for fault detection.

In this paper, a new methodology dealing with the problem of detecting and characterizing small leaks in diesel air paths is developed. To achieve this goal, a new scheme based on a neural network technique is proposed. The nominal mode (without leaks) and leakage mode corresponding to several diameters of leaks were trained using a Levenberg-Marquardt algorithm. Before using the acquired data, a feature selection task is proposed in order to reduce the complexity of the problem. The main challenge of the proposed approach is the use of selected sensors leading to a reduced cost. The data for the considered modes are generated using the xMOD platform which will be described later.

The paper is organized in this way. First, the considered problem is presented in Section 2. Section 3 describes the proposed approach in detail. A brief description of neural networks based on the steepest-descent and Gauss-Newton methods is given, and the main detection and characterization scheme is illustrated. After a brief description of the MOD tool used in engine data collecting, Section 4 gives some results obtained using our approach. Then, these results are discussed and commented in order to illustrate the effectiveness of leakage detection and characterization.

\section{Problem Statement}

Over the past several years, anti-pollution standards have become more stringent and then the constraints for the automotive industry have also become very complex. The main objective of these standards is to reduce the emission levels of cars. In the case of diesel engines, there are several pollutants: carbon monoxide, unburned hydrocarbons, nitrogen oxides (NOx) and diesel particulate matter. Usually, the emission level proportionally increases with the appearance of faults in diesel engines, more precisely in diesel air paths. These faults can be due to sensor failures, actuator failures or system degradation. In this paper, the latter failure class is considered. More precisely, the leakage detection and characterization in diesel air paths is studied. This failure can cause multiple non-desired system behaviors. In addition to the high emission levels, this failure causes multiple non-desired effects such as: 
- Changes in the operating points of the air path subsystems,

- Incomplete combustion in cylinders,

- Appearance of smoke and the reduction of performance.

Often, this type of failure can be confused with the two other types of faults, i.e., sensors or actuators; consequently, it is very important to distinguish this fault from others.

In addition to the main objective of this paper, the feature selection problem is considered. It is well known that today's vehicles are characterized by increased complexity due to the growing number of embedded sensors. Consequently, the use of selected subsets of sensor data which are in correlation with the considered problem is widely desired in such applications.

In this work, our main objective is to detect and characterize air leaks in diesel air paths regardless of their diameters. Before performing leak detection and characterization, we carry out a feature selection in order to reduce the data complexity.

It is important to specify that, for this application, small leaks are hidden and are very difficult to detect because of the phenomenon of the non-solicitation of the system.

\section{Proposed Approach}

Nowadays, the neural network is an essential tool used in many research activities for complex industrial systems. An advantage of using neural networks to detect system faults is that they can interpret the measurement data. Indeed, a neural network has both the ability to generalize an obtained model and, to apply the associative property to the available memory. The error tolerance, characterizing the neural network, effectively deals with the errors of the model. In addition, it can perform nonlinear mapping and also learn dynamic behaviors in order to generalize the obtained models.

Generally, the collected data for the detection and characterization process are noisy, but, the error tolerance ability of neural networks enables the detection scheme to differentiate the pattern from noise. This property is a huge advantage in fault detection and isolation problem resolution. In addition, similar patterns are separated using the property of characterization of a neural network.

Leak detection in intake systems is very difficult to achieve especially when the operating point corresponds to low load-torque couple. In these conditions, the compressor in the air path is not solicited by the driver, leading to similar pressure between the intake system and the atmosphere. This constraint requires an improved detection and characterization algorithm. The proposed system must increase the accuracy of the model, enhance the performances of the vehicle and guarantee the management of small leaks. In this paper, the Levenberg-Marquardt (LM) algorithm is proposed to carry out the detection and characterization tasks. The LM algorithm is used to train the air path diesel dynamics. Once the dynamics are modeled, the leak is detected and characterized by comparing the new measurements with the model established using a neural network. The proposed approach contains two blocks which are the training block and the decision block. This approach is shown in Figure 1. 


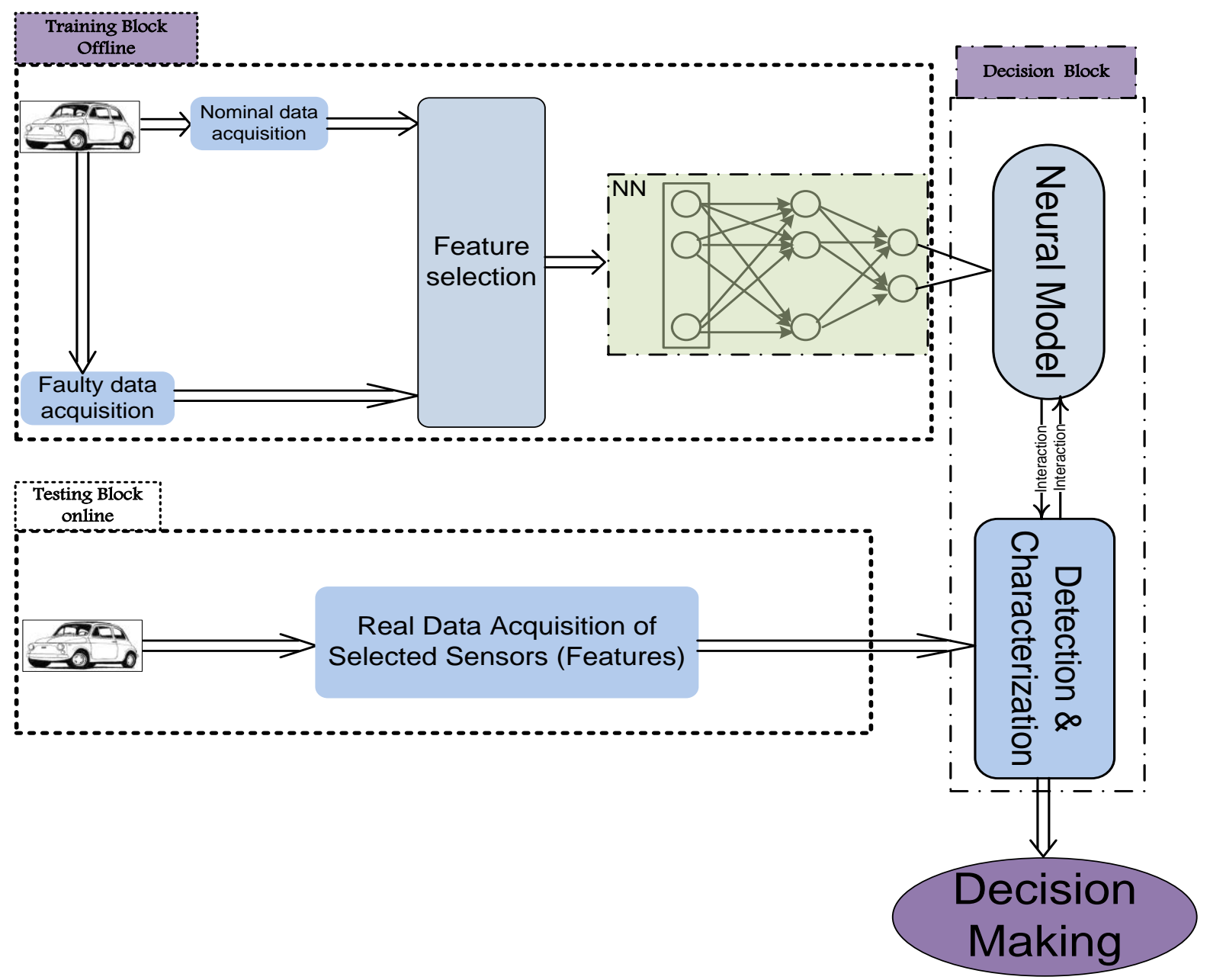

Figure 1. Detection and characterization scheme.

The proposed approach is designed to operate in on-line mode, thus, a classic process of real data acquisition is adopted in this work. It is important to remember that in this application we use only sensors selected by the feature selection algorithm and summarize the intake behavior of the diesel air path. The data acquired in this step are sent to the decision block in order to detect and characterize the leaks affecting the vehicle.

\subsection{Training Block}

\subsubsection{Feature Selection}

In this work, a very popular feature selection is chosen; it is the Chi-square algorithm [20]. Chi-square is a simple and general algorithm which achieves feature ranking using a discretization process. This algorithm is combined with a neural network classifier to select the features that we must keep.

The Chi-square algorithm is based on the $X^{2}$ which runs in two stages in this manner:

- Stage 1:

1. Set the sigLevel to 0.5 for all features;

2. Sort each feature according to its values;

3. Compute the $X^{2}$ value for every pair of adjacent intervals, such that: 


$$
\begin{gathered}
X^{2}=\sum_{i=1}^{2} \sum_{j=1}^{k} \frac{\left(A_{i j}-E_{i j}\right)^{2}}{E_{i j}} \\
\text { with }: E_{i j}=R_{i} * C_{j} / N
\end{gathered}
$$

where:

$k$ : number of classes;

$A_{i j}$ : number of samples in the $i^{\text {th }}$ interval and $j^{\text {th }}$ class;

$R_{i}$ : number of samples in the $i^{\text {th }}$ interval;

$C_{j}$ : number of samples in the $j^{\text {th }}$ class;

$N$ : total number of samples;

4. Merge the pair of adjacent intervals with the lowest $X^{2}$ value until the $X^{2}$ value of each pair of adjacent intervals exceeds sigLevel.

- Stage 2:

1. Start with the sigLevelO corresponding to the last sigLevel value determined in the first stage;

2. Associate sigLevel $(i)$ with each feature and run merging;

3. Consistency test:

If inconsistency $<\delta$ merge intervals and decrease sigLevel $(i)$;

Else, eliminate the $i^{\text {th }}$ features for the next step.

Firstly, the WEKA [21] data-mining tool is used to perform Chi-square ranking. The features are sorted according to their rank. Secondly, the most important features are selected using the neural network classifier, which will be described later. More precisely, the features will be eliminated iteratively from least important to most important and the weight of the eliminated features is evaluated according to the obtained classification Mean Squared Error (MSE).

\subsubsection{Training}

Pattern classification using neural networks consists of determining the class boundaries using the classifier. The training phase of neural networks achieves this goal. In this paper, a gradient-based training algorithm is used. This category of algorithm is most commonly used by researchers. One of these algorithms is Hessian-based algorithms; they can significantly reduce the convergence time. The Levenberg-Marquardt algorithm [22] belongs to category of Hessian-based techniques; it makes use of the advantages of Hessian-based algorithms in the optimization of nonlinear least squares.

The Levenberg-Marquardt algorithm is a well-known optimization technique. It locates the minimum of a function which is expressed by the sum of squares of nonlinear functions. This algorithm, widely used in many disciplines, is a combination of the Steepest-Descent and the Gauss-Newton methods. Depending on the distance between the current position and the best one, these techniques operate intermittently; if the current position is far from the best one the steepest-Descent is applied, otherwise the Gauss-Newton takes over. The Steepest-Descent technique used in the LM algorithm is slow, but it guarantees the convergence property. When the current position approaches the best one, the LM algorithm switches to the Gauss-Newton method which converges rapidly.

For neural network training, the objective function is the error of the type: 


$$
M S E=\frac{1}{2} \sum_{k=1}^{p} \sum_{i=1}^{n_{0}}\left(y_{k l}-a_{k l}\right)^{2}
$$

where $y_{k l}$ are real data of diesel engines, $a_{k l}$ are a network output, $p$ is the total number of samples and no represents the total number of nodes in the output layer.

In this work, the neural network used contains five layers. The first layer is the input layer which receives the data corresponding to the selected sensors which are used in this application. The three following layers are the hidden ones which represent the network core. The last one is the output layer which generates two signals when the detection task is considered or four signals when the tasks of detection and characterization are considered together.

The steps required in the neural network using the LM algorithm in batch-mode training are the following:

- Compute the corresponding network outputs and evaluate the mean square error for all inputs as in Equation (1);

- Calculate the Jacobian matrix $j(x)$, where $x$ represents the weights and biases of the network;

- Solve the equation which adapts weights in order to obtain $\Delta x$, The update of the weighted vector $\Delta x$ is computed as follows:

$$
\Delta x=\left[J^{T}(x) J(x)+\mu I\right]^{-1} J^{T}(x) R
$$

where $\mu$ is the training parameter and $\mathrm{R}$ is a vector of size pn0 computed as follows:

$$
R=\left(\begin{array}{c}
y_{11}-a_{11} \\
y_{12}-a_{12} \\
\cdots \\
y_{21}-a_{21} \\
y_{22}-a_{22} \\
\cdots \\
y_{p n_{0}}-a_{p n_{0}}
\end{array}\right) E_{i j}=R_{i} * C_{j} / N
$$

$J^{T}(x) J(x)$ is referred to as the Hessian matrix.

- Recalculate the error using $x+\Delta x$. If there is the reduction of the error calculated in step 1 , the training parameter $\mu$ is reduced by $\mu^{-}, \operatorname{keep} x=x+\Delta x$ and return to step 1 . If there is no reduction, increase $\mu$ by $\mu^{+}$and go back to step 3. $\mu^{+}$and $\mu^{-}$are fixed by the user;

- The algorithm is stopped in two cases; when the gradient is less than the predefined value, or when the error is below a given error objective.

Generally, the training step in neural networks is very complex and requires considerable computing resources, especially in on-line cases. In this work, the training problem is carried out in off-line mode, then, the obtained neural model is used to detect and characterize leakage in on-line mode. The adopted neural network returns both the nominal behavior corresponding to the system without leakage and the faulty system behavior (occurrence of leakage). 


\subsection{Decision Block}

The decision block is the most essential component of the proposed scheme where the leaks in the intake of the air path are detected and characterized using the neural model developed in the training step. Direct interactions are established between the detection and characterization block with the neural network model in order to estimate the actual state of the system. The decision block works in two modes, "detection mode" or "detection and characterization mode". If the detection mode is considered, the decision block returns the two possible outputs, "No Leakage" or "Leakage". In "detection and characterization mode", it returns four outputs which are, "No Leak", "Low Leakage", "Medium Leakage" and "High Leakage".

\section{Application}

A critical operating mode system is considered to illustrate the effectiveness of the proposed approach. This mode concerns the case of low engine load, speed and couple, where the leak detection and characterization problem is not systematically realized. In this application, the data acquisition is carried out using xMOD software.

\section{1. $x M O D$ Software}

xMOD is a software platform that was developed at "IFP Energies Nouvelles" combining heterogeneous models and a virtual experimentation laboratory. These heterogeneous models are generated by different simulation tools, such as Matlab/Simulink, AMESim, Dymola, SimulationX and GT Power. A combination of these tools means benefiting from the advantages of each modeling and simulation tool, and the user can freely select these tools.

In this work, xMOD is used to simulate the diesel engine functioning, especially the air path behavior. The simulation model produced by IFP "Energies Nouvelles" is used, to which a leak model has been added. The diameter of the leak can be freely adjusted. The simulation results can be recovered and stored in text files.

\subsection{MSE: All Features vs. Selected Features}

Before presenting the results with selected features, a comparison of the MSE evaluation for "all features" and "selected features" is presented in Table 1.

In order to illustrate the advantage of feature selection, the MSE values are jointly shown with their training run times. In this table, we can firstly observe that MSE values corresponding to the use of all features are greater than the MSE values when only the selected features are used. Secondly, we observe that the run time corresponding to the use of all features is always higher than when the selected features are used. For example, when the torque value is set to $40 \mathrm{Nm}$, all MSE values of the all features case are greater than those corresponding to the selected features case. The same conclusion can be drawn for the remaining three cases except for some values. The detection and characterization tasks are presented for the selected features case. 
Table 1. Mean Squared Error (MSE) Evolution with torque variation.

\begin{tabular}{|c|c|c|c|c|c|c|c|c|}
\hline \multirow{2}{*}{$\begin{array}{l}\text { Torque } \\
\text { Leaks }\end{array}$} & \multicolumn{2}{|c|}{40 Nm (MSE/Run Time) } & \multicolumn{2}{|c|}{110 Nm (MSE/Run Time) } & \multicolumn{2}{|c|}{130 Nm (MSE/ Run Time) } & \multicolumn{2}{|c|}{150 Nm (MSE/Run Time) } \\
\hline & & $\begin{array}{l}\text { Selected } \\
\text { Features }\end{array}$ & & $\begin{array}{l}\text { Selected } \\
\text { Features }\end{array}$ & & $\begin{array}{l}\text { Selected } \\
\text { Features }\end{array}$ & & $\begin{array}{l}\text { Selected } \\
\text { Features }\end{array}$ \\
\hline $0.1 \mathrm{~mm}$ & $0.208 / 14^{\prime} 59^{\prime \prime}$ & $0.0221 / 10^{\prime} 35^{\prime \prime}$ & $0.168 / 12^{\prime} 40^{\prime \prime}$ & $0.0166 / 10^{\prime} 41^{\prime \prime}$ & $0.167 / 14^{\prime} 50^{\prime \prime}$ & $0.0109 / 8^{\prime} 40^{\prime \prime}$ & 0.00547/15'06" & 0.00799/8'59" \\
\hline $0.2 \mathrm{~mm}$ & $0.0449 / 16^{\prime} 29^{\prime \prime}$ & $0.0189 / 8^{\prime} 49^{\prime \prime}$ & $0.00790 / 12: 44$ & 0147/8'55" & $0.00988 / 13^{\prime} 10^{\prime \prime}$ & $0.0163 / 8^{\prime} 45^{\prime \prime}$ & $0.00083 / 15^{\prime} 47^{\prime \prime}$ & $0.000812 / 10^{\prime} 15^{\prime \prime}$ \\
\hline $0.3 \mathrm{~mm}$ & $0.0270 / 18^{\prime} 44^{\prime \prime}$ & $0.0114 / 9^{\prime} 19^{\prime \prime}$ & $0.00608 / 12^{\prime} 52^{\prime \prime}$ & $0.00871 / 9^{\prime} 44^{\prime \prime}$ & $0.00298 / 16^{\prime} 23^{\prime \prime}$ & $0.00266 / 8^{\prime} 45^{\prime \prime}$ & $0.00430 / 14^{\prime} 17^{\prime \prime}$ & $0.00165 / 10^{\prime} 03^{\prime \prime}$ \\
\hline $0.4 \mathrm{~mm}$ & $0.0205 / 15^{\prime} 24^{\prime \prime}$ & $0.0204 / 9^{\prime} 25^{\prime \prime}$ & $0.00239 / 14^{\prime} 39^{\prime \prime}$ & 0.00413/10'15" & $0.00219 / 13^{\prime} 55^{\prime \prime}$ & $0.00401 / 10^{\prime} 53^{\prime \prime}$ & $0.00273 / 13^{\prime 2} 23^{\prime \prime}$ & $0.00419 / 11^{\prime} 38^{\prime \prime}$ \\
\hline $0.5 \mathrm{~mm}$ & $0.0186 / 18^{\prime} 41^{\prime \prime}$ & $0.0127 / 8^{\prime} 34^{\prime \prime}$ & $0.00458 / 14^{\prime} 02^{\prime \prime}$ & $0.00369 / 7^{\prime} 57^{\prime \prime}$ & $0.00156 / 12^{\prime 22} 2^{\prime \prime}$ & $0.00222 / 10^{\prime 2} 23^{\prime \prime}$ & $0.00208 / 13^{\prime} 44^{\prime \prime}$ & $0.00428 / 11^{\prime} 25^{\prime \prime}$ \\
\hline $0.6 \mathrm{~mm}$ & $0.0121 / 15^{\prime} 50^{\prime \prime}$ & $0.00807 / 9^{\prime} 30^{\prime \prime}$ & $0.00447 / 14^{\prime} 17^{\prime \prime}$ & $0.00315 / 9^{\prime} 52^{\prime \prime}$ & $0.00351 / 14^{\prime} 12^{\prime \prime}$ & $0.00104 / 9^{\prime} 34^{\prime \prime}$ & $0.00656 / 15^{\prime} 00^{\prime \prime}$ & $0.00221 / 11^{\prime} 04^{\prime \prime}$ \\
\hline $0.7 \mathrm{~mm}$ & $0.0180 / 15^{\prime} 57^{\prime \prime}$ & $0.00404 / 9^{\prime} 43^{\prime \prime}$ & $0.00669 / 16^{\prime} 11^{\prime \prime}$ & $0.00234 / 9^{\prime} 07^{\prime \prime}$ & $0.00100 / 8^{\prime} 02^{\prime \prime}$ & $0.00102 / 8^{\prime} 40^{\prime \prime}$ & $0.00601 / 14^{\prime} 33^{\prime \prime}$ & $0.000873 / 10^{\prime} 01^{\prime \prime}$ \\
\hline $0.8 \mathrm{~mm}$ & $0.00867 / 17^{\prime} 26^{\prime \prime}$ & $0.00727 / 8^{\prime} 21^{\prime \prime}$ & $0.00584 / 13^{\prime} 42^{\prime \prime}$ & $0.00198 / 9^{\prime} 54^{\prime \prime}$ & $0.00099 / 7^{\prime} 18^{\prime \prime}$ & $0.00101 / 9^{\prime} 56^{\prime \prime}$ & $0.000897 / 13^{\prime} 35^{\prime \prime}$ & 0.00193/9'52" \\
\hline $0.9 \mathrm{~mm}$ & $0.00618 / 14^{\prime} 30^{\prime \prime}$ & $0.00910 / 8^{\prime} 56^{\prime \prime}$ & $0.00451 / 13^{\prime} 32^{\prime \prime}$ & $0.00415 / 9^{\prime} 33^{\prime \prime}$ & $0.00211 / 13^{\prime} 28^{\prime \prime}$ & $0.00131 / 8^{\prime} 58^{\prime \prime}$ & $0.00270 / 13^{\prime} 36^{\prime \prime}$ & $0.00087 / 11^{\prime} 29^{\prime \prime}$ \\
\hline $1.0 \mathrm{~mm}$ & 0.00989/13'11" & $0.00638 / 9^{\prime} 14^{\prime \prime}$ & $0.00217 / 15^{\prime} 01^{\prime \prime}$ & $0.00111 / 9^{\prime} 43^{\prime \prime}$ & $0.00102 / 13^{\prime} 10^{\prime \prime}$ & $0.00099 / 5^{\prime} 09^{\prime \prime}$ & $0.00392 / 13^{\prime} 15^{\prime \prime}$ & $0.000896 / 8^{\prime} 41^{\prime \prime}$ \\
\hline
\end{tabular}

\subsection{Detection Task Results}

The first property of the proposed approach is the detection ability. In this situation, the neural network trains two classes: "No Leakage mode" and "Leakage mode". The training set consists of 10,000 samples without leaks and 10,000 samples with leaks. We choose three values of leaks, $0.1 \mathrm{~mm}$, $0.4 \mathrm{~mm}$ and $0.9 \mathrm{~mm}$. These results are obtained using only selected features in the training algorithm.

\section{Interpretation}

Figures 2-10 show the effectiveness of the proposed approach where we can see that the leak is detected for all considered diameters. Mean Squared Error (MSE) values give information about the accuracy of the neural network used. From the obtained results we can first remark that the MSE values increase when the torque values decrease. For example, in the first case (Figures 2-4) when the leak diameter is set to $0.1 \mathrm{~mm}$, the MSE value decreases from $0.0240(2.4 \%)$ to $0.00746(0.7 \%)$ when the torque increases from $110 \mathrm{Nm}$ to $150 \mathrm{Nm}$. This observation can be explained by the fact that the air path system (compressor) works at a lower speed. In other words, in low speed, the mechanical compressor of the air path is not solicited. The same remark is applied to cases 2 and 3.

Naturally, the leak is easily detected when it is large, but, it becomes extremely difficult to detect when it is very small. The obtained results show that the proposed approach is efficient and the leak is detected in all cases even when it is equal to $0.1 \mathrm{~mm}$ (almost negligible leakage). In addition, this approach gives better results at higher engine load, speed and couple which is expected due to higher flow on the air intake system. 
Case1: Leak $=0.1 \mathrm{~mm}$

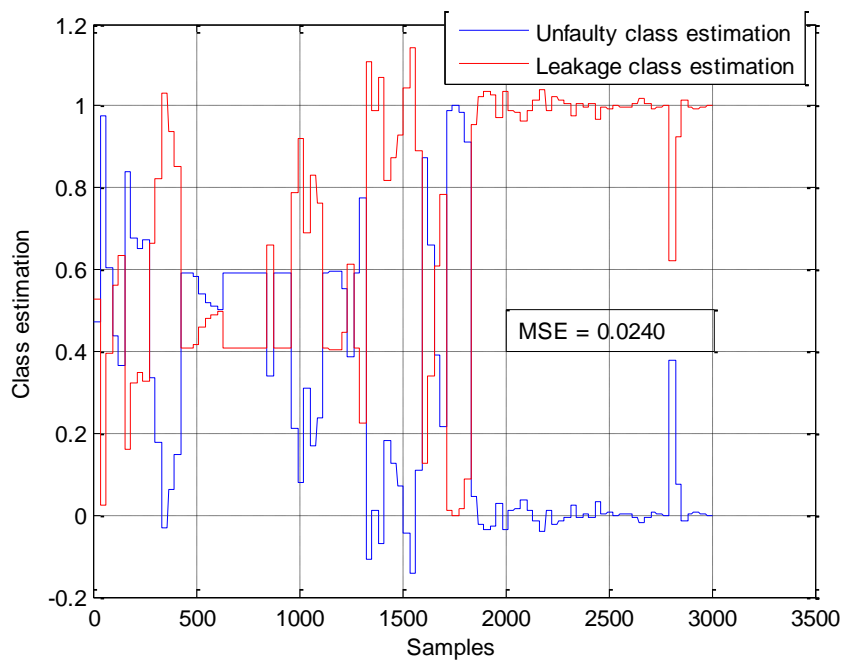

Figure 2. Engine_speed $=1000 \mathrm{rpm}$ and torque $=110 \mathrm{Nm}$.

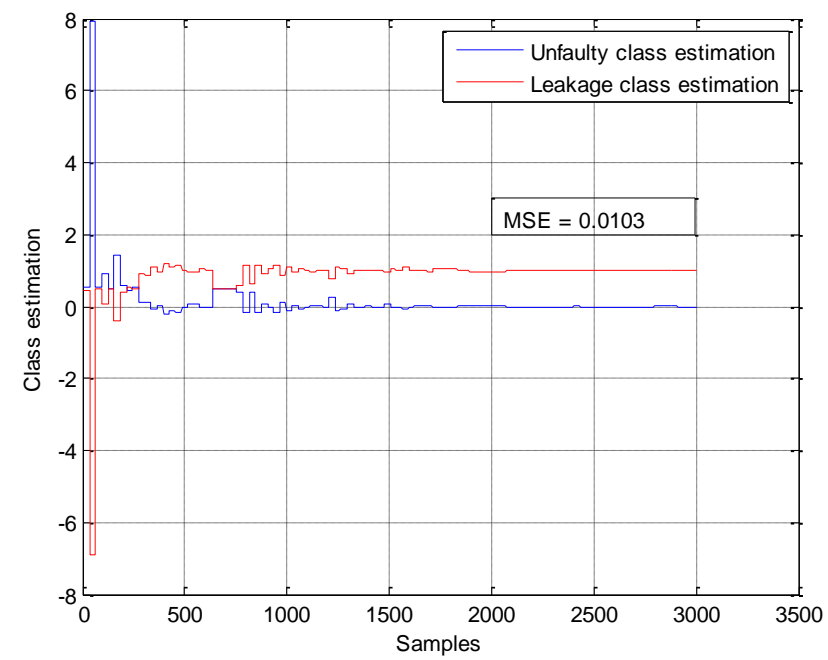

Figure 3. Engine_speed $=1000 \mathrm{rpm}$ and torque $=130 \mathrm{Nm}$.

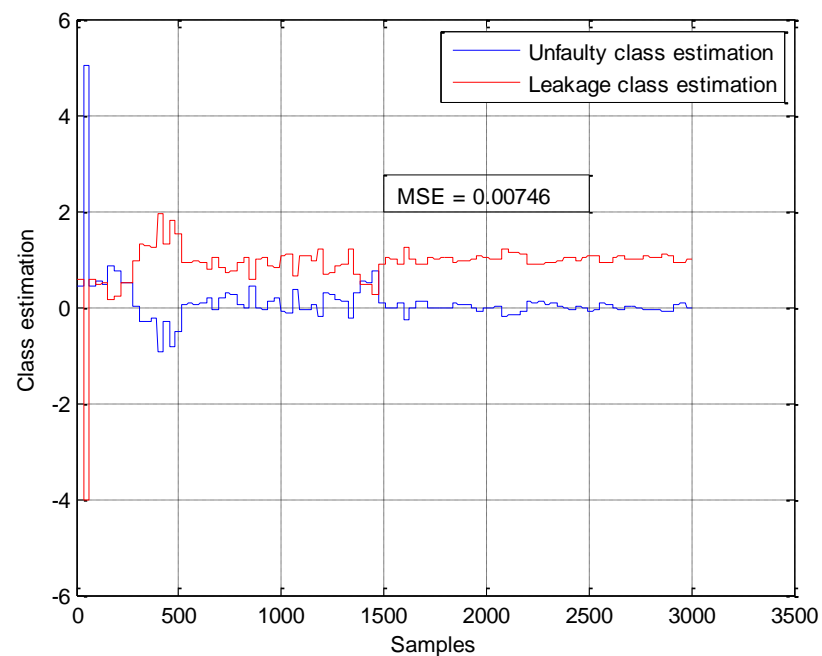

Figure 4. Engine_speed $=1000 \mathrm{rpm}$ and torque $=150 \mathrm{Nm}$. 
Case2: Leak $=0.4 \mathrm{~mm}$

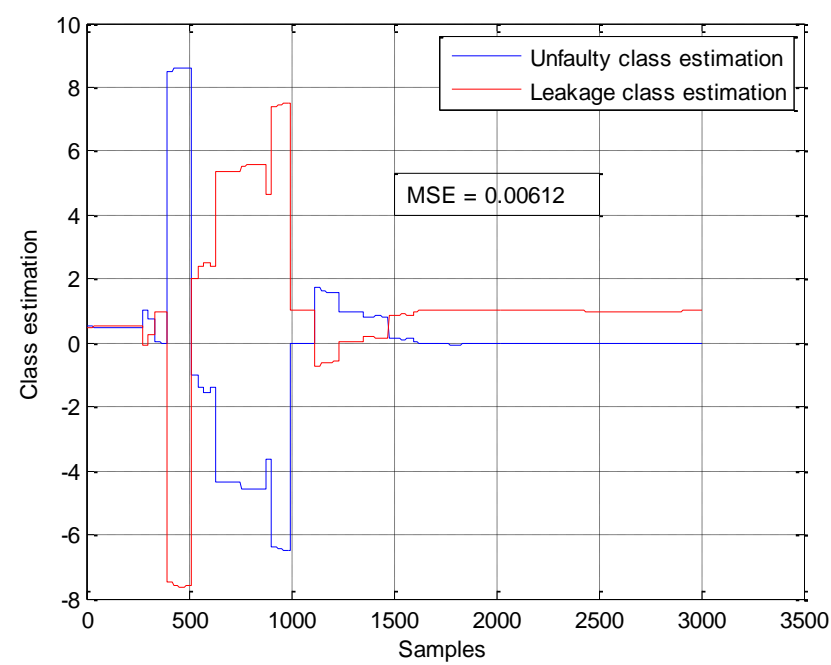

Figure 5. Engine_speed $=1000 \mathrm{rpm}$ and torque $=110 \mathrm{Nm}$.

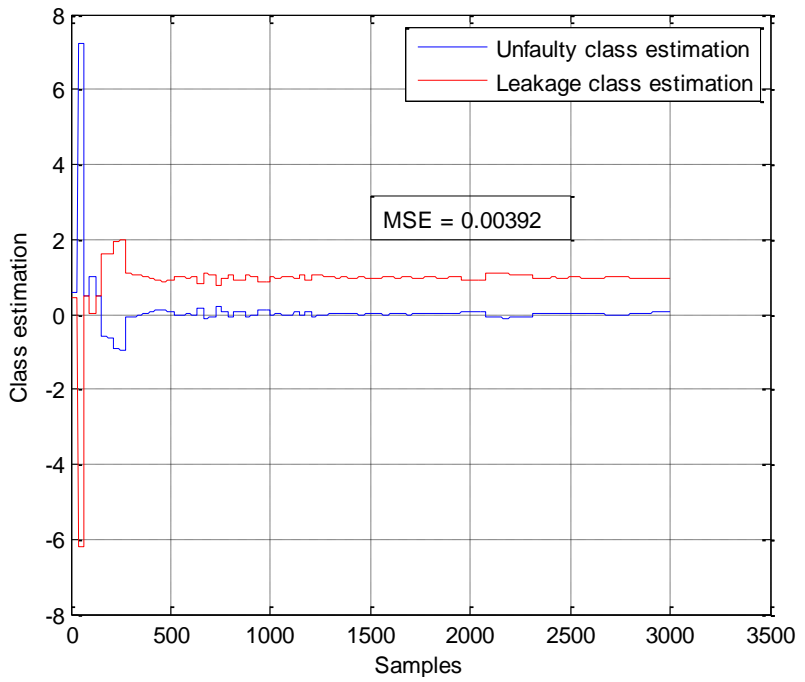

Figure 6. Engine_speed $=1000 \mathrm{rpm}$ and torque $=130 \mathrm{Nm}$.

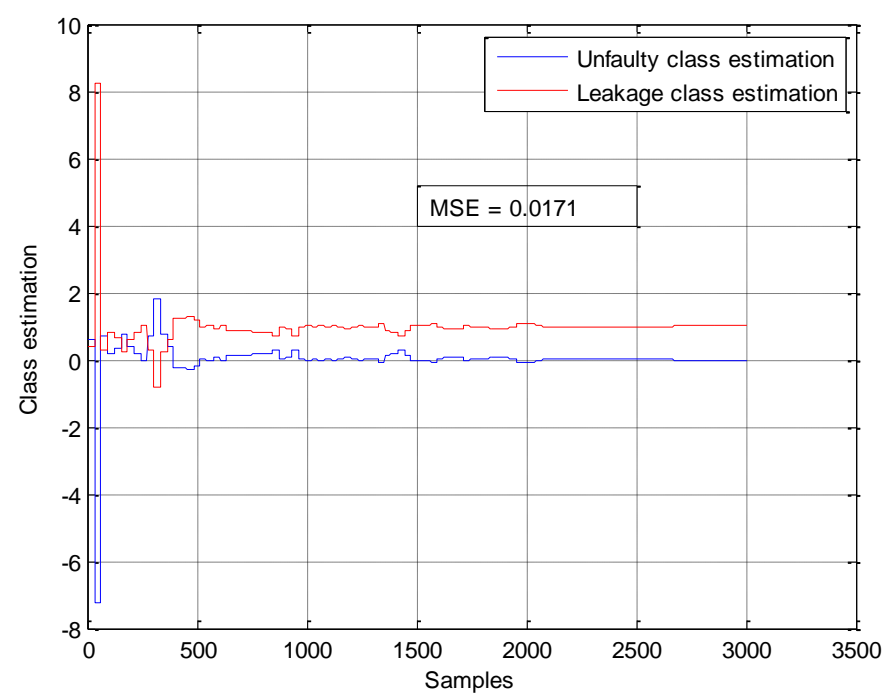

Figure 7. Engine_speed $=1000 \mathrm{rpm}$ and torque $=150 \mathrm{Nm}$. 
Case3: Leak $=0.9 \mathrm{~mm}$

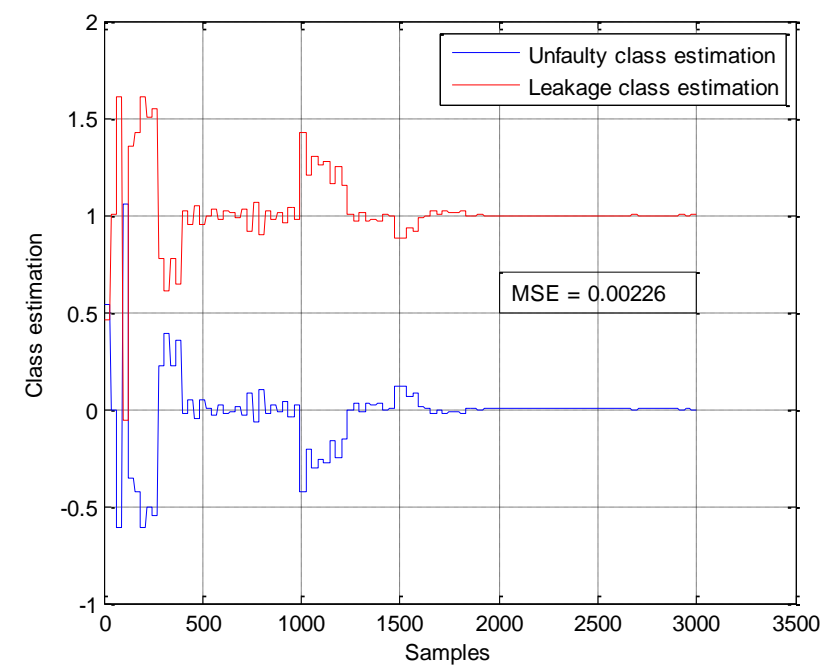

Figure 8. Engine_speed $=1000 \mathrm{rpm}$ and torque $=110 \mathrm{Nm}$.

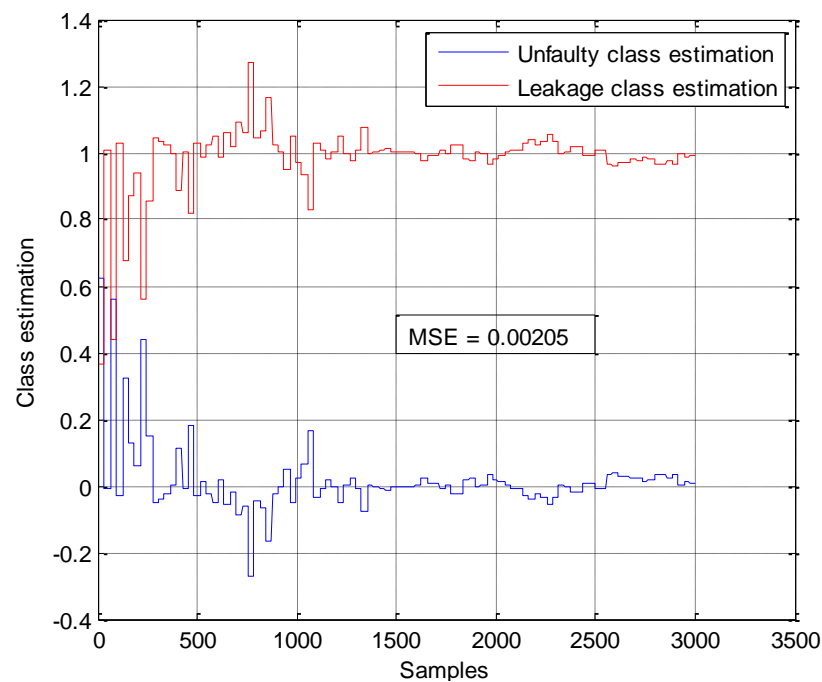

Figure 9. Engine_speed $=1000 \mathrm{rpm}$ and torque $=130 \mathrm{Nm}$.

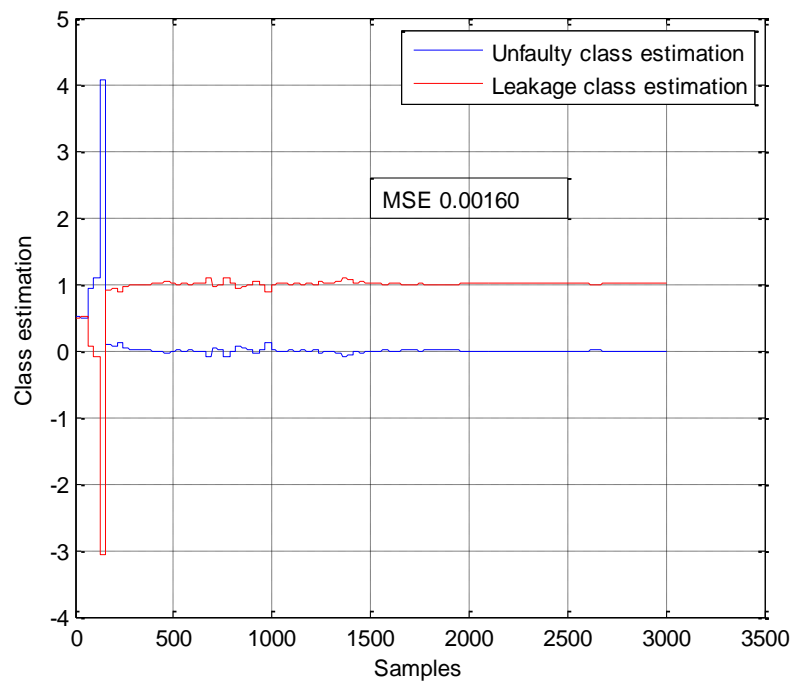

Figure 10. Engine_speed $=1000 \mathrm{rpm}$ and torque $=150 \mathrm{Nm}$. 


\section{Characterization Task Results}

This property is very important and allows for the estimation of the severity of the leak. Thus, the characterization of the leak diameter is highly desired and it is often difficult to accomplish. In order to do this, the neural network is trained with the data of four modes which are: "No Leak", "Low Leakage", "Medium Leakage" and "High Leakage". The three last modes respectively correspond to "Leak = $0.1 \mathrm{~mm}$ ", "Leak $=0.4 \mathrm{~mm}$ " and "Leak $=0.9 \mathrm{~mm}$ ". The data of each mode contains 10,000 samples. The Figures 11-13 show the leak characterization in each mode.

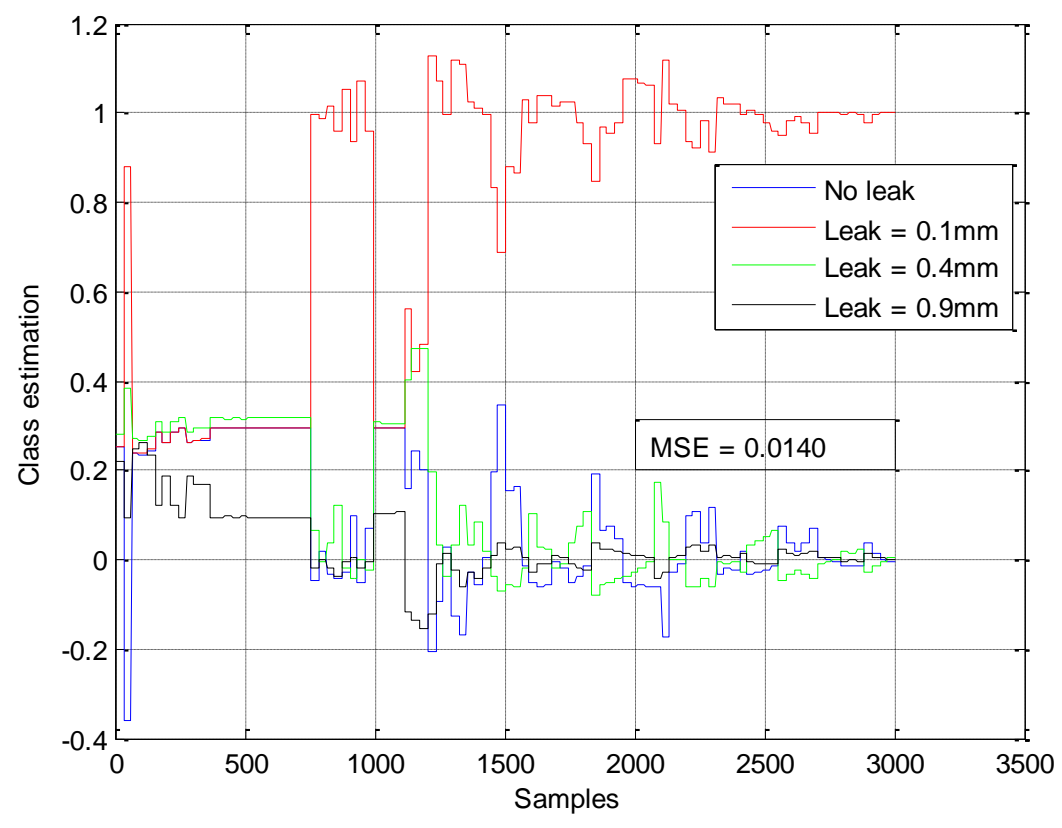

Figure 11. Leak Characterization in "Low Leakage" case.

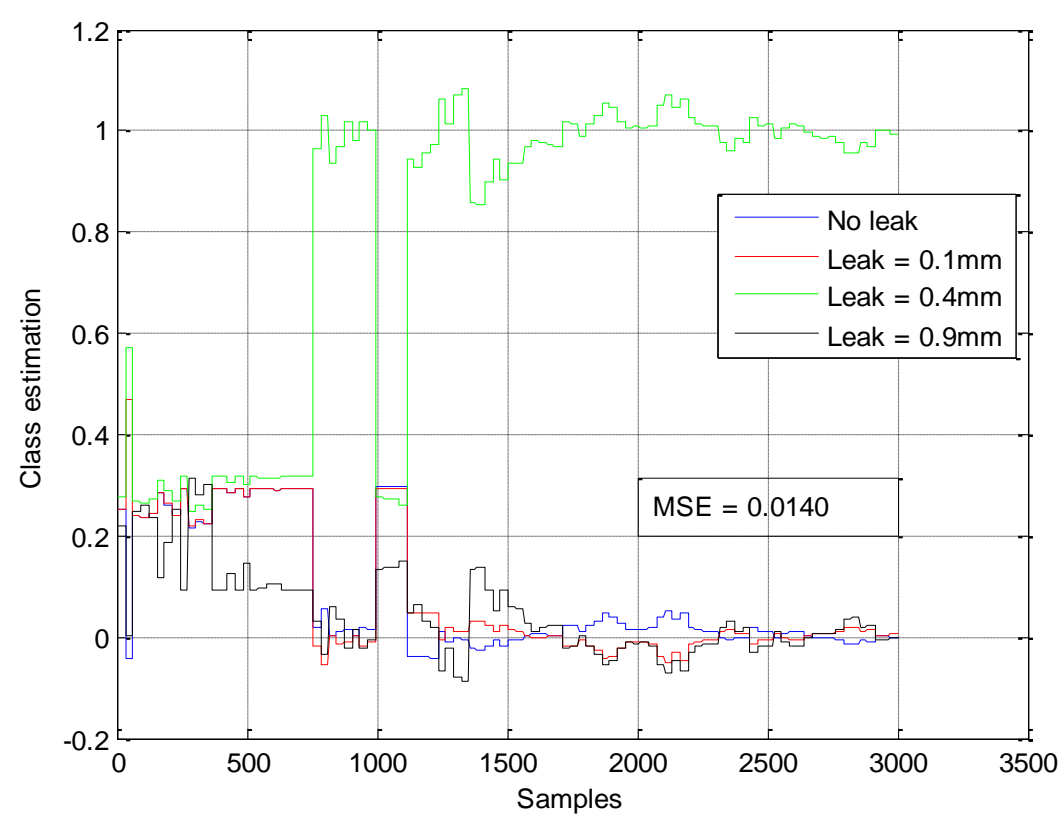

Figure 12. Leak Characterization in "Medium Leakage" case. 


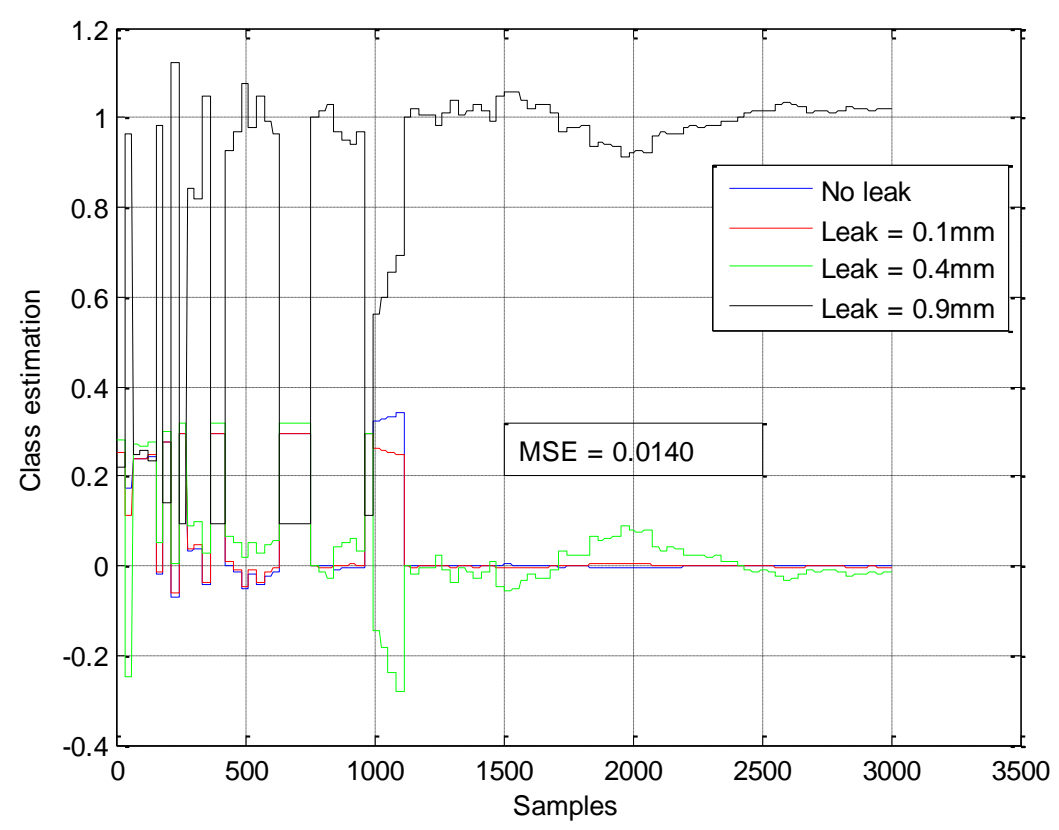

Figure 13. Leak Characterization in "High Leakage" case.

These figures show the effectiveness of the proposed approach dealing with leak characterization. Firstly, we remark that MSE value (0.0140) is low and the accuracy of the neural network is demonstrated. Secondly, the leakage class is found for each case.

\section{Conclusions}

A leak detection and characterization approach for diesel air paths has been developed. The proposed approach contains two blocks: a training block and a decision block. The first one is realized off-line and combines a feature selection algorithm with a neural network based on the Levenberg-Marquardt optimization. The L-M function was chosen for its accuracy and adaptability; it combines two different techniques according to the current position of the solution compared to the best one. The second block uses the neural model obtained in training phase in order to detect and characterize leaks that appear in the air path system. The detection and characterization capability is evaluated using the MSE index.

The proposed approach effectively solves the leak detection and characterization problem, especially in the case of small leaks in critical operating points (low speed and torque). In order to validate this solution, the proposed algorithms will be implemented in a real diesel engine.

\section{Acknowledgments}

The authors gratefully thank the ANR (Agence Nationale de la Recherche), "Haute Normandie" Region and FEDER for financially supporting this work.

\section{Author Contributions}

This paper proposes a model-free diagnosis approach for leak detection and characterization in Diesel Engines. The main contribution in this work is the capacity of the proposed approach to detect a very small leak in the air path equal to $0.1 \mathrm{~mm}$ in diameter. The minimum leak detected with other approaches 
is equal to $4 \mathrm{~mm}$ in diameter [23]. The proposed approach also gives good results for leak characterization in static mode. Other investigations are in progress for the characterization task in dynamical cases and for sensor fault diagnosis.

Ghaleb Hoblos was the scientific responsible of this work within the ANR project DIVAS "Diesel Innovative VVA and Advanced air System for Downspeeding". Ghaleb Hoblos and Mourad Benkaci conceived and designed the proposed approach. Mourad Benkaci performed most of the tests. Ghaleb Hoblos and Mourad Benkaci wrote the paper.

\section{Conflicts of Interest}

The authors declare no conflict of interest.

\section{References}

1. Nyberg, M.; Nielsen, L. Model-based diagnosis for the air intake system of the SI-engine. In Proceedings of the SAE International Congress and Exposition, Detroit, MI, USA, 24-27 February 1997.

2. Nyberg, M. Model-based diagnosis of an automotive engine using several types of fault models. IEEE Trans. Control Syst. Technol. 2002, 10, 679-689.

3. Nyberg, M. Model-based diagnosis of the air path of an automotive diesel engine. Control Eng. Pract. 2004, 12, 513-525.

4. Gertler, J.; Costin, M.; Fang, X.; Kowalczuk, Z.; Kunwer M.; Monajemy, R. Model-based diagnosis for automotive engine-Algorithm development and testing on a production vehicle. IEEE Trans. Control Syst. Technol. 1995, 13, 61-69.

5. Isermann, R. Process fault detection based on modeling and estimation methods: A survey. Automatica 1984, 20, 387-404.

6. Sorsa, T.; Koivo, H.N. Application of artificial neural networks in process fault diagnosis. Automatica 1993, 29, 843-849.

7. Capriglione, D.; Liguori, C.; Pianese C.; Pietrosanto, A. On line sensor fault detection, isolation and accommodation in automotive engines. IEEE Trans. Instrum. Meas. 2003, 52, 1182-1189.

8. Sangha, M.S.; Yu, D.L.; Gomm, J.B. On-Board monitoring and diagnosis for spark ignition air path via adaptive neural networks. J. Automob. Eng. 2006, 220, 1641-1655.

9. Chen, P.C. A novel diagnostic system for gasoline-engine leakage detection. J. Automob. Eng. 2011, 225, 225-685.

10. Christina, B.V; Tshilidzi, M. Application of Feature Selection and Fuzzy ARTMAP to Intrusion Detection. In Proceedings of the IEEE International Conference on Systems, Man and Cybernetics, Taipei, Taiwan, 8-11 October 2006.

11. Carpenter, G.A.; Grossberg, S.; Markuzon, N.; Reynolds, J.H.; Rosen, D.B. Fuzzv ARTMAP: A Neural Network Architecture for Incremental Supervised Learning of Analog Multidimentional Maps. IEEE Trans. Neural Netw. 1992, 3, 698-713.

12. Wang, L.; Yu, J. Fault Feature Selection Based on Modified Binary PSO with Mutation and its Application in Chemical Process Fault diagnosis. In Proceedings of the International Conference on Advanced in Natural Computation N1, Changsha, China, 27-29 August 2005; pp. 832-840. 
13. Xu, Z.; Xuan, J.; Shi, T.; Hu, Y. Application of a modified fuzzy ARTMAP with feature-weight learrning for the fault diagnosis of bearing. Expert Syst. Appl. 2009, 36, 9961-9968.

14. Sugumaran, V.; Muralidharan, V.; Ramachandran, K.I. Feature selection using Decision Tree and classification through Proximal Support Vector Machine for fault diagnostics of roller bearing. Mech. Syst. Signal Process. 2007, 21, 930-942.

15. Casimir, R.; Boutleux, E.; Clerc, G.; Yahoui, A. The use of features selection and nearest neighbors rule for faults diagnostic in induction motors. Eng. Appl. Artif. Intell. 2006, 19, 169-177.

16. Chiang, L.H.; Pell, R.J. Genetic algorithms combined with discriminant analysis for key variable identification. J. Process Control 2004, 14, 143-155.

17. Verron, S.; Tiplica, T.; Kobi, A. Fault detection and identification with a new feature selection based on mutual information. J. Process Control 2008, 18, 479-490.

18. Mao, Y.; Xia, Z.; Yin, Z.; Sun, Y.; Wan, Z. Fault Diagnosis Based on Fuzzy Support Vector Machine with Parameter Tuning and Feature Selection. Chin. J. Chem. Eng. 2007, 15, 233-239.

19. Wang, K. Neural Network Approach to Vibration Feature Selection and Multiple Fault Detection for Mechanical Systems. In Proceedings of the ICICIC'06 First International Conference on Innovative Computing, Information and Control, Beijing, China, 30 August-1 September 2006; Volume 3, pp. 233-239.

20. Alexandrov, M.; Gelbukh, A.; Lozovo, G. Chi-square Classifier for Document Categorization. In Proceedings of the 2nd International Conference on Intelligent Text Processing and Computational Linguistics, Mexico City, Mexico, 18-24 February 2001.

21. Witten, I.H.; Eibe, F. Data Mining: Practical Machine Learning Tools and Techniques, 2nd ed.; Morgan Kaufmann Publishers Inc.: San Francisco, CA, USA, 2005.

22. Levenberg, K. A Method for the Solution of Certain Non-linear Problems in Least Squares. Q. Appl. Math. 1944, 2, 164-168.

23. Ceccarelli, R.; Canudas-de-Wit, C.; Moulin, P.; Sciarretta A. Model-based Adaptive Observers for Intake Leakage Detection in Diesel Engines. In Proceedings of the IEEE ACC'09 American Control Conference, Saint Louis, MO, USA, 10-12 June 2009.

(C) 2015 by the authors; licensee MDPI, Basel, Switzerland. This article is an open access article distributed under the terms and conditions of the Creative Commons Attribution license (http://creativecommons.org/licenses/by/4.0/). 